This PDF is a selection from an out-of-print volume from the National Bureau of Economic Research

Volume Title: Medical Care Output and Productivity

Volume Author/Editor: David M. Cutler and Ernst R. Berndt, editors

Volume Publisher: University of Chicago Press

Volume ISBN: 0-226-13226-9

Volume URL: http://www.nber.org/books/cut101-1

Publication Date: January 2001

Chapter Title: Hedonic Analysis of Arthritis Drugs

Chapter Author: Iain M. Cockburn, Aslam H. Anis

Chapter URL: http://www.nber.org/chapters/c7637

Chapter pages in book: (p. 439 - 462) 


\title{
Hedonic Analysis of Arthritis Drugs
}

\author{
Iain M. Cockburn and Aslam H. Anis
}

\subsection{Introduction}

This study examines the market for a group of drugs used to treat rheumatoid arthritis (RA) during the period 1980-92. Rheumatoid arthritis is a painful, debilitating, and progressive disease which affects millions of people worldwide, with very substantial effects on health and the economy. Regrettably, in contrast to some other major health problems such as heart disease, depression, ulcers, and bacterial infections, this is an area where therapeutic innovations have thus far had comparatively little impact on physicians' ability to reverse the disease. RA currently has no "cure" and the effectiveness of available treatments is limited. Compared to other drug classes the rate of new product introductions has been slow, and, at the time of writing, there have been no breakthroughs of the same order of significance as the discovery and development of SSR Is for treatment of depression, $\mathrm{H}_{2}$ antagonists for ulcers, or ACE inhibitors for hypertension.

Nonetheless, the market for RA drugs is far from static. There have been significant changes over the past fifteen years in the market shares of competing products. Interestingly, relative prices have changed relatively

Iain M. Cockburn is professor of finance and economics at Boston University and a research associate of the National Bureau of Economic Research. Aslam H. Anis is associate professor of health economics in the Department of Health Care and Epidemiology, University of British Columbia, and team leader of health economics at the Center for Health Evaluation and Outcome Sciences, St. Paul's Hospital, Vancouver.

The authors thank Ernst Berndt, Zvi Griliches, John Esdaile, and NBER seminar participants for helpful comments, and Jennifer Anderson and David Felson for access to their databases on safety/efficacy profiles. The authors are grateful to BC Pharmacare for access to claims data; BEA, NBER, and Eli Lilly for financial support; and Merck for access to library records. Sophia Wang provided invaluable and very competent research assistance. The authors take full responsibility for any remaining errors. 
little, and these market dynamics appear to be driven primarily by other factors. Here we focus on the role played by publication of clinical research findings. In contrast to traditional hedonic analysis where product characteristics are fixed but new products incorporating different quality levels appear over time, here the set of products is fixed while their measured quality changes over time. New information about the relative efficacy and toxicity of existing drugs accumulates through the publication of clinical trial results, and this information appears to have had a significant impact on the pattern of drug use.

A number of clinical aspects of rheumatoid arthritis are important structural features of the market for drugs used to treat the disease. We therefore begin with a brief review of the nature of RA and its treatment. We then discuss issues related to the measurement of the relative efficacy and toxicity of drug treatments for RA. Next, we present economic data on the market for a specific set of drugs used in the treatment of severe RA and consider them in the context of models of demand for differentiated products. We then report the results of estimating price and market share equations. In the concluding section, we suggest alternative approaches that may provide some additional insight, in particular analysis of the role of advertising and promotional expenditures.

\subsection{Rheumatoid Arthritis}

RA is one of the most prevalent diseases affecting joints and connective tissue. RA is an autoimmune disease: For reasons that are still poorly understood, the body's immune system begins to malfunction, attacking healthy tissue. Like related conditions such as lupus erythematosus, psoriatic arthritis, and scleroderma, the disease is systemic and chronic. Tissues are affected throughout the body, and although some patients experience prolonged periods of remission, most are affected for a lifetime. ${ }^{1}$

RA is characterized by inflammation of the synovium (a membrane which lines the joints) resulting in stiffness, pain, warmth, and swelling in joints. As the disease progresses, inflamed cells release an enzyme which erodes surrounding bone and cartilage, resulting in increased pain, loss of movement, and eventually destruction of the joint. ${ }^{2}$ Patients experience greater and greater pain and loss of mobility. Fatigue often accompanies the "classical" joint symptoms. In late stages of the disease, skin and vascular problems (such as leg ulcers) may develop, along with damage to eyes and nerves and inflammation of lymph nodes, heart, and lungs.

1. Brewerton (1994) gives a comprehensive and readable overview of arthritis and its treatment. See also Cash and Klippel (1994), Wolfe (1990) and Steinman (1993).

2. Establishing a conclusive diagnosis of RA can be difficult, especially in its early stages, since it shares many symptoms with other autoimmune diseases. Note that RA should not be confused with osteoarthritis, an even more prevalent disease, which has a distinct clinical profile and disease process. 
Research into the fundamental causes of the disease has inconclusively investigated many factors ranging from endocrine disorders to nutrition, geography, psychological conditions, and occupational hazards. Current thinking suggests that some infectious agent may trigger the damaging autoimmune response in persons who have a genetic predisposition. However, while a specific genetic marker (HLA-DR4) has been found to be present in a large fraction of RA patients, not all patients have the marker, and only a small fraction of people who have the marker go on to develop RA. Neither has the proposed infectious agent (possibly an unknown virus) been identified, though various other arthritic and rheumatic conditions have been associated with infection by a number of organisms such as borellia (the Lyme disease spirochete) and some streptococcal bacteria.

RA affects between 1 and 2 percent of the population of OECD countries. Women are two to three times more likely than men to develop disease. In adults the onset of the disease is typically between ages forty and sixty, though significant numbers of people experience severe symptoms in their thirties and forties, and the disease can occur at any age. In some patients deterioration is rapid, while in others the disease progresses very slowly. Once affected, the outlook for most patients is poor. In many cases patients experience temporary relief of symptoms, but only very few have a complete remission of the disease. Chronic severe pain and restricted mobility have a very significant impact on the quality of life of RA patients. Even with aggressive drug therapy, 7 percent of RA patients are significantly disabled within five years, and 50 percent are too disabled to work ten years after the onset of the disease. In addition to the morbidity effects of RA, Pincus and Callahan (1993) estimate that life expectancy is reduced among patients with RA by at least ten years.

By any measure the total burden of the disease is substantial. Qualityadjusted life years (QALYs) lost may be as many as seven million per year in the United States. ${ }^{3}$ The combination of severe health impact, widespread incidence, and relatively early onset mean that very substantial economic losses are attributable to RA. For example, in 1997 the Arthritis Foundation reported that musculoskeletal conditions such as RA cost the U.S. economy approximately $\$ 65$ billion per year in direct expenses and lost output.

\subsubsection{Treatment Options for Rheumatoid Arthritis}

Over the course of the disease, medical treatment of RA patients consists of physical intervention and drug therapy. Counseling or other psy-

3. In Canada, RA occurs in approximately 1 percent of the population, or about 270,000 people. It has been estimated that the average Canadian has significant pain and/or disability from arthritis resulting in an average of 2.5 quality-adjusted life years (QALYs) lost. See Torrance and Feeny (1989) and Reynolds et al. (1993). Since RA tends to be more frequently disabling than osteoarthritis, a conservative estimate of the total disability among Canadians from RA would hence be 675,000 QALYs lost. 
chotherapeutic intervention may also play an important role in helping patients cope with the impact of the disease, and many patients also turn to "alternative" medicine. Physical intervention takes the form of physical therapy directed toward preservation of joint function and surgical procedures to address severe pathologies of specific joints (e.g., hip replacement). Drug treatment, the focus of this study, is given to almost all patients who consult a physician: Of the approximately 5.1 million patient visits per year in the United States where RA is a primary diagnosis, more than 90 percent involved one or more drugs' being prescribed.

Two principal classes of drugs are used to treat RA: nonsteroidal antiinflammatory drugs (NSAIDs) and disease-modifying antirheumatic drugs (DMARDs). These two classes account for more than 65 percent of all prescriptions to RA patients, with corticosteroids accounting for a further 19 percent. It is important to note that drug therapy for RA normally follows a treatment hierarchy: Drug treatment begins with NSAIDs and moves on to DMARDs as the disease progresses.

NSAIDs are the most frequently prescribed drugs for RA. Large numbers of drugs fall into the NSAID class; among the most commonly used are aspirin, ibuprofen (Motrin), naproxen (Naprosyn), diclofenac (Voltaren), and piroxicam (Feldene). NSAIDs reduce inflammation and have an analgesic effect but do not affect progression of the disease. NSAIDs act quickly and are well tolerated by many patients but can cause a number of dangerous side effects, particularly when used in the high dosages indicated for RA. Gastrointestinal bleeding is the most frequently encountered severe side effect. ${ }^{4}$ While NSAIDs are the first line of defense, they offer only palliative treatment of symptoms, and as the disease progresses patients will typically be given one of the DMARDs. This does not usually imply discontinuation of NSAID therapy, and in fact between 80 and 90 percent of patients are prescribed drugs from both classes.

The second-line DMARDs can suppress symptoms and slow the progress of the disease, though they cannot halt it. DMARDs are slow acting, taking weeks or months before any significant improvement is noticed by the patient, and are often poorly tolerated. Different drugs are used with varying degrees of success in different patients. Furthermore, many patients are forced to discontinue the drug because of serious side effects. Minor, though uncomfortable, side effects such as dermatitis, nausea, and mouth ulcers are quite frequently experienced. The incidence of serious side effects such as retinal damage, renal failure, liver damage, and reduction in blood cell counts, while uncommon, nonetheless requires close medical supervision and frequent diagnostic testing.

4. COX-2 inhibitors, a new class of NSAIDs with a more selective mechanism of action and lower incidence of side effects, have recently been introduced into the U.S. market. These drugs include celecoxib (Celebrex) and rofecoxib (Vioxx). 


\begin{tabular}{|c|c|c|c|c|}
\hline Drug & $\begin{array}{c}\text { Brand } \\
\text { Name(s) }\end{array}$ & $\begin{array}{l}\text { U.S. Market } \\
\text { Intro }\end{array}$ & Other Indications & Manufacturer \\
\hline Auranofin & Ridaura & 1985 & & SKB \\
\hline Azathioprine & Imuran & 1968 & $\begin{array}{l}\text { Immune suppression } \\
\text { for transplants }\end{array}$ & $\begin{array}{l}\text { Glaxo } \\
\quad \text { Wellcome }\end{array}$ \\
\hline $\begin{array}{l}\text { Gold sodium } \\
\text { thiomalate }\end{array}$ & Myochrysine & $<1980$ & & Merck \\
\hline Aurothioglucose & Solganal & $1989 ?$ & & Schering \\
\hline \multirow{2}{*}{$\begin{array}{l}\text { Hydroxychloroquine } \\
\text { methotrexate }\end{array}$} & Plaquenil & 1956 & Malaria & Winthrop \\
\hline & Rheumatrex & 1955 & Leukemia, psoriasis & $\begin{array}{l}\text { Lederle, } \\
\text { generics }\end{array}$ \\
\hline D-penicillamine & Cuprimine & 1963 & Chelation & $\begin{array}{l}\text { Merck, } \\
\text { Wallace }\end{array}$ \\
\hline Sulfasalazine & Azulfidine & 1952 & $\begin{array}{l}\text { Ulcerative colitis, } \\
\text { Crohn's disease }\end{array}$ & $\begin{array}{l}\text { Kabi, } \\
\quad \text { generics }\end{array}$ \\
\hline
\end{tabular}

The DMARDs approved for treatment of RA during the period of this study are listed in table 11.1. One point to note from this table is that many of these drugs are quite old, having been first introduced to the market many years ago. Auranofin (Ridaura) was the only strictly new molecule approved for RA in the period covered by this study. Other products such as methotrexate are new to the market in the sense that they have recently gained regulatory approval for treatment of RA, though they may have been approved for other indications for many years or may have been used informally or in research settings for treatment of RA. (Lederle introduced Rheumatrex, a formulation of methotrexate specifically targeted at the RA market, in 1986.) Sulfasalazine, methotrexate, and the antimalarials are off-patent, but generic production is significant only for methotrexate. It is also important to note that the original or primary indication of most of the drugs was not RA. With the exception of the gold compounds, the activity of the DMARDs against RA was discovered subsequent to their first introduction to the market. Methotrexate was an early treatment for cancer, while hydroxychloroquine was developed as an antimalarial. The precise mechanism of action of most of these drugs is not well understood, though most have their therapeutic effect through suppressing the immune response. The anti-inflammatory activity of gold compounds appears to be specific to arthritic conditions, while the immunosuppressant activity of azathioprine and methotrexate is much more general.

In addition to DMARDs, physicians may also prescribe corticosteroids. This occurs in about 20 percent of patient visits in the United States. While these drugs can often produce dramatic short-term improvement in symptoms, their long-term use is limited by serious side effects, principally osteoporosis and increased susceptibility to infections. As a last resort, pa- 
tients may also be prescribed highly toxic third-line immunosuppressant drugs such as cyclophosphamide, cyclosporine, or chlorambucil. Without a new therapy which induces a lasting remission, physicians face difficult decisions and trade-offs in drug therapy for RA. ${ }^{5}$

The timing of moving a patient from well-tolerated NSAIDs to the more toxic DMARDs is controversial, with some physicians arguing for early and aggressive second-line therapy to preempt irreversible joint damage, despite serious side effects. Even within the DMARD class it is far from clear which drug to prescribe. Only a fraction of patients obtain significant benefit from any one agent and even then the effect is often short-lived, typically lasting for only a few months or years. Over the twenty- to thirtyyear course of the disease, a patient will typically cycle through a series of therapeutic alternatives as their physician attempts to arrest, or often merely to minimize, the cumulative destruction wrought by the disease. Furthermore, professional opinion has changed over time regarding which drugs to use, and when. The information base on the relative efficacy and toxicity of these agents continues to evolve as new scientific evidence from clinical trials is published and physicians individually and collectively accumulate more experience. The efficacy/toxicity trade-off lies at the heart of the prescribing decision, and changing perceptions of where drugs are located in this space drives our analysis of demand for these drugs.

\subsection{Measuring the "Quality" of Drug Treatments for RA}

We attempt to measure the characteristics of different DMARD drugs in two general dimensions: efficacy and toxicity. Unlike some previous work on hedonics of pharmaceutical products we pay little attention to differences in the dosage regimen. Though characteristics such as the number of times a day the patient must take the drug appear to be an important determinant of the relative value of different ulcer drugs and antidepressants (see Suslow 1992, 1996 and Berndt, Cockburn, and Griliches 1997), we believe them to be much less important here. The very close involvement of the physician and the severe nature of the disease suggest to us that the impact of dosing regimens on patient compliance is unlikely to be an important factor. ${ }^{6}$

5. Leflunomide (Arava), approved by the FDA in September 1998, is a new DMARD with a novel mechanism of action and potentially less severe side effects. A number of experimental drugs, largely from the biotech sector of the industry, hold some promise for significant progress in treating arthritis and other autoimmune inflammatory disorders. Infliximab (Enbrel), a genetically engineered protein, was approved by the FDA for treatment of RA in late 1998, but of most of these "large molecule" drugs are still in the early stages of testing. See Wall Street Journal, 17 July 1997, B1.

6. As a practical matter, dosage regimens for these drugs vary widely, are difficult to compare directly, and often involve complicated "ramp-up" schedules paced over many weeks. For example, the maintenance dose of methotrexate is $7.5 \mathrm{mg}$ spread over a week, while 
Our primary measures of efficacy and toxicity are computed from the reported results of published clinical trials. We assume that the best available information about the relative efficacy and toxicity of substitute drugs comes from published reports of clinical trials that appear in peerreviewed scientific journals. These reports constitute a longitudinal data set which tracks the evolution of information on each drug over time.

Based on Felson, Anderson, and Meenan (1990), we begin with the universe of 216 published trials published between 1966 and 1995 listed in the MEDLINE database. Protocols and methodology vary widely across trials, and to establish a basis for comparison of results across trials (and to maintain a minimal level of methodological quality) papers were excluded if they did not meet the following criteria:

Patient profile: adults eighteen and older, meeting American Rheumatism Association diagnostic criteria for RA

Random assignment to treatment groups

Blinded trial (at a minimum single-blinded)

Appropriate minimum dosage levels

At least eight weeks' duration

Imposing these criteria resulted in all but 66 of the original set of published trials being excluded.

\subsubsection{Efficacy Measures}

Efficacy of drugs in these trials is established by compiling measurements of a number of standard physiologic markers and outcome measures for patients in the different treatment groups at the beginning and end of each trial. These were

Erythrocyte sedimentation rate (ESR), which is a physiologic marker of the level of overall systemic inflammation, derived from testing blood samples drawn from trial participants at predefined intervals during the trial

Tender joint count (TJC), which is a measure of the extent and severity of the disease in terms of the number of affected joints, compiled according to a standard protocol by a physician or nurse who assesses the patient and measured as difference (or percentage difference) over baseline

Grip strength (GS), which is another measure of the extent and severity of the disease, performed by measuring the pressure the patient is able to exert on a standard mechanical device, captured as either mean percent-

suifasalazine must be taken in relatively large amounts several times per day, and most of the gold compounds are injectable. Quantifying dosage regimens with variables measuring route of administration, dosage frequency, and so on is tantamount econometrrically to simply using drug dummies. 
age improvement over baseline or the mean improvement standardized by baseline standard deviation

Apart from these measurements, efficacy can also be measured by the reported rate at which patients dropped out of each trial due to "lack of efficacy."

\subsubsection{Toxicity Measures}

Toxicity is much harder to measure consistently. We have not been able to assemble consistent data on the actual incidence of side effects in each trial. Following previous work we have experimented with variables constructed by counting the number of side effects listed under categories such as "severe" or "frequent" in standard reference sources, or constructing dummy variables reflecting the locus of specific side effects (kidney damage, central nervous system, retina, etc.) but these perform poorly in experimental regressions. ${ }^{7}$ Our preferred measure of toxicity is the reported rate at which patients dropped out of clinical trials due to "toxicity." Summary statistics for these variables are given in table 11.2.

\subsubsection{Changes in Quality over Time}

Because new trials are conducted periodically, information accumulates steadily over time, and variables constructed from reported trial results form a longitudinal data set. We combine data from different trials in a variety of ways intended to capture the evolution over time of the scientific information available to prescribing physicians.

One possibility is to simply assign a value to each variable in each year based on the most recently published study. Thus we "ratchet" the level of each variable up or down in each year that a new trial came out, and carry forward the previous value otherwise. (In tables below we refer to these measures as "latest.")

A second approach is to do a "rolling" cumulative meta-analysis which pools treatment groups over time and across drugs. As new trials are published results for each group of patients are added to the previous total, resulting in a continuously expanding sample. Mean treatment effects are the weighted sum of treatment effects in all trials to date.

Third, we modify the cumulative meta-analysis by imposing various schemes of declining weights over time to capture "depreciation" of knowledge. We expect the results of trials conducted many years in the past to weigh less heavily upon current prescribing practice than more recent evidence. The simplest such weighting scheme is a three- or fiveyear moving average. Alternatives such as a perpetual inventory deprecia-

7. Clinicians may be most strongly influenced by the relative incidence of severe adverse reactions. We have not yet compiled data on these effects. But note that since these events are very rare, their probability of occurrence is difficult to measure precisely. 


\begin{tabular}{lrrrrr}
\hline & \multicolumn{5}{c}{ Sample Means } \\
\cline { 2 - 5 } & \multicolumn{4}{c}{ Efficacy } & Toxicity \\
\cline { 2 - 5 } Drug Name and Daily Dose & TJC & GS & ESR & Dropout & Price $^{\text {a }}$ \\
\hline Auranofin, 6mg & 8.44 & 26.98 & 10.79 & 0.16 & 1.91 \\
Azathioprine, 100mg & 9.78 & 33.11 & 13.73 & 0.27 & 1.67 \\
Gold salts, 7mg & 9.15 & 38.20 & 10.79 & 0.40 & 1.07 \\
Antimalarials, 400mg & 9.21 & 39.89 & 11.41 & 0.04 & 1.42 \\
Methotrexate, 12.5mg & 13.23 & 33.11 & 13.49 & 0.16 & 1.31 \\
D-penicillamine, 600mg & 8.78 & 37.26 & 22.65 & 0.33 & 1.70 \\
Sulfasalazine, 2.5g & 12.28 & 28.53 & 20.64 & 0.37 & 0.84 \\
Placebo, n.a. & 4.80 & 9.74 & 1.26 & 0.07 & n.a. \\
\hline
\end{tabular}

Note: n.a. $=$ not available.

"1992 U.S. dollars per daily maintenance dose.

tion scheme or fixed declining weights do not yield materially different results.

\subsection{Model}

The theoretical literature provides little guidance on the appropriate functional form for estimating quality-adjusted prices. Following many previous hedonic pricing studies (for pharmaceutical products, see Suslow's analysis of ulcer drugs [1996] or Berndt, Cockburn, and Griliches's work on antidepressants [1997]) we use a semilog reduced form:

$$
\ln \left(p_{j t}\right)=x_{j t} \beta+Z \gamma+\varepsilon_{j \prime},
$$

where $x_{j,}$ represents the measured quality (i.e., toxicity and efficacy) characteristics of drug $j ; j=1, \ldots, J$ at time $t ; Z_{t}$ is a set of time dummies; and $p_{\mu}$ denotes the time series of prices for $\operatorname{drug} j$.

For the market share equation, we follow Berry (1994) and Berry, Levinsohn, and Pakes (1995) in specifying a logit type discrete choice model of demand for differentiated products to analyze the DMARD market. See King (1996) for a successful application of a modification of this approach to the anti-ulcer market. Following Berry we postulate that the utility of consumer $i$ for product $j$ is given by the function $U\left(x_{j}, \xi_{j}, p_{j}, \Theta_{d}, v_{i}\right)$, where $x_{j}, \xi_{j}, p_{j}, \Theta_{d}$ are observed product characteristics, unobserved product characteristics, and price and demand parameters, respectively. The term $v_{i}$ is unobserved by the econometrician and represents a consumer-specific component of utility. To implement the model, one has to make specific parametric assumptions about the consumer-specific variables, analogous to the choice of functional form for a homogenous good demand equation. The utility derived by consumer $i$ for product $j$ can be written as 


$$
u_{i j}=x_{i j} \beta_{i}-\alpha p_{j}+\xi_{i j}+\varepsilon_{i j} .
$$

Averaging over consumers (we assume that the physicians who exercise control over the drug consumption decision act as perfect agents for their patients) and introducing time subscripts to reflect the fact that the perceived safety and efficacy characteristics of drugs change over time, we obtain a mean consumer utility level from choosing $\operatorname{drug} j$ at time $t$ as

$$
\delta_{j t}=x_{j t} \beta-\alpha p_{j}+\xi_{j t},
$$

where $\xi_{j t}$ may be interpreted as the mean of consumers'/physicians' valuations of an unobserved product characteristic that is not captured by $x_{j t}$ and we use the assumption that $E\left[\varepsilon_{i j}\right]=0 .{ }^{8}$

In addition to the competing DMARDs, $j=1, \ldots, J$, we also assume the existence of an outside good $j_{0}$ with price $p_{0}$. In this context, consumption of the outside good can be thought of as the quantities of NSAIDs and all other non-DMARDs consumed by RA patients. (Empirically, almost all RA patients' visits to doctors result in their being prescribed either an NSAID or a DMARD or both. Only a tiny number of patients receive no drug therapy.) Letting $q_{j}$ and $q_{j}$ and $q_{0}$ denote the quantities of $\operatorname{drug} j$ and the outside good, respectively, market shares for $\operatorname{drug} j$ are just $s_{j t}=q_{j t} /\left(q_{j t}+q-{ }_{j t}+q_{0 t}\right)$.

In this model it is assumed that all aspects of market demand are completely determined by the mean utility level $\delta_{j t}$ and, without going into the specifics of supply side dynamics and alternative characterizations of market equilibrium, we adopt the special case of the logit model to solve for mean utility levels as a function of observed market shares. Given the utility function in equation (2), if $\beta_{i}=\beta$ for all consumers $i$, and $\varepsilon_{i j}$ is an iid variable which follows the type I extreme value distribution, then market share of $\operatorname{drug} j$ is given by the logit formula

$$
s_{j t}\left(\delta_{j t}\right)=\exp \left(\delta_{j t}\right) / \exp \left(\delta_{0 t}+\Sigma \delta_{j t}\right) .
$$

By substitution and by normalizing the mean utility of the outside good to equal zero, we get the following linear model for market shares:

$$
\ln \left(s_{j t}\right)-\ln \left(s_{0 t}\right)=\delta_{j t}=x_{j t} \beta-\alpha p_{j}+\xi_{j t},
$$

where $s_{j t}$ and $p_{j t}$ are the quantity share and price of the $j$ th DMARD at time $t$. The unobserved characteristics of the drug, $\xi_{j t}$, becomes the error term.

In our implementation of this basic estimating equation we deflate prices by the BLS producer price index for pharmaceuticals to remove the general trend of inflation. (This is equivalent to a slightly different

8. See Berry (1994) for more on possible ways of decomposing $\beta_{i}$ and on the assumptions that yield invariant $\alpha$ and $\beta$ across individuals. 
specification of equation [2] with a normalization of the utility level of the outside good which leaves the price of the outside good in the estimating equation.) As argued below, we believe prices to be largely exogenous to this market and are therefore unconcerned about endogeneity of this variable. Given the panel structure of the data, we can address the issue of potential correlation between $\xi_{j t}$ and the other explanatory variables by including a fixed drug effect, so that $\xi_{j t}=\mu_{j}+\eta_{j t}$ with $\eta_{j t}$ assumed to have the usual desirable properties.

\subsection{Price and Quantity Data}

Econometric analysis of the market for DMARDs requires basic data on prices and quantities of these drugs sold, and careful attention to the definition of the RA market. Our primary data on prices and quantities for the DMARDs are drawn from reports of wholesale transactions in the United States published by IMS America Inc., a market research company. IMS collects information on revenues and quantities of individual drug products by wholesale distributors at a very fine level of detail; for example, 100-mg tablets, 100-count bottle. (We have also collected data on retail transactions in British Columbia which were reimbursed under the province's Pharmacare program. The Pharmacare program is universal and covers all residents with varying levels of coverage depending on sociodemographic status. Trends in these data match the U.S. wholesale market very closely.)

A major difficulty with these kinds of data, however, is that they are collected by drug product, not by disease indication. As pointed out above, many of the DMARDs have multiple uses, and in fact their primary use may be for quite different medical problems. Analyzing demand for these drugs for treatment of RA requires that we distinguish between these uses. This may not be important for measuring prices: Lacking some means to discriminate among consumers through packaging or reformulation it is not unreasonable to assume that one price holds for all sales of a particular formulation of a drug regardless of the intended use. This is likely to be particularly true for the wholesale market. By contrast, in measuring quantities it is vitally important to distinguish between markets in the sense of different medical conditions. Large (and varying) amounts of these drugs are used for treatment of other diseases.

Figure 11.1 presents series on U.S. wholesale prices for DMARDs for the period 1980-92. Prices are measured in dollars per daily dose unit. Daily doses are the "typical maintenance dose" taken from a number of standard reference publications such as the Physician's Desk Reference. It should be noted that the dosage given to any particular patient may vary substantially from the amounts we use here: Treatment of most patients may involve considerable experimentation with dosages. Some of the drugs 


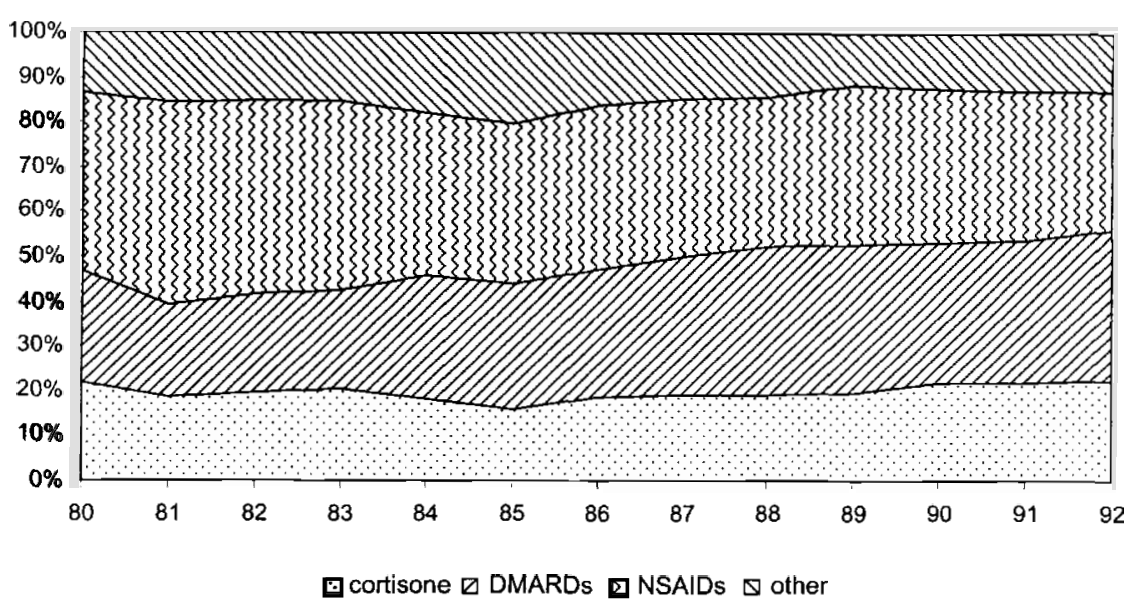

Fig. 11.1 Drug class shares of NDTI mentions for RA

also have a fairly complicated "ramp-up" dosage regime lasting many weeks before the maintenance dose is treated. Relative prices based on the cost of initiating drug therapy and maintaining it for a total of three months are very similar to the daily dose prices presented here.

Perhaps the most striking feature of figure 11.1 is that prices are so similar across the major products and move so closely together. Over time prices rise steadily with general inflation, with few significant changes relative to one another. The major exception is sulfasalazine, whose roughly constant nominal price corresponds to a sustained decline - in real terms, a steady fall. Methotrexate's price rises relatively steeply during the mid1980s, driven largely by the introduction of Lederle's branded Rheumatrex product, while the rate of increase in the price of the injectable gold products moderates somewhat toward the end of the period.

To address the market definition problem, we need information on the fraction of each drug's consumption which is specifically for the treatment of RA. For this we turn to another IMS publication, the National Drug and Therapeutic Index (NDTI). The NDTI reports results from surveying a sample of physicians. Each physician is asked to supply various pieces of information about patient visits; for our purposes the most useful data are the reports on primary diagnosis and which drugs (if any) were prescribed. In these reports a "drug mention" is equivalent to one prescription. IMS imputes figures for the total U.S. population from the survey sample and provides tabulations by drug and by diagnosis. Thus for each drug we can compute a breakdown of prescriptions by diagnosis, and for the diagnosis of RA, a breakdown of prescriptions by drug. These data provide valuable insight into market dynamics.

Table 11.3 summarizes information on consumption of DMARDs by 
diagnosis, reporting the percentage of prescriptions of each drug for which RA was the primary diagnosis. This fraction is high and stable for some drugs such as injectable gold salts and auranofin, indicating that their principal market is indeed RA. For other DMARDs, such as sulfasalazine and azathioprine, the "outside" uses are very substantial, averaging more than 75 percent of prescriptions. Furthermore, there are significant changes in these fractions over time. The fraction of d-penicillamine used for RA falls from 91 percent in 1980 to 72 percent in 1992, while the same statistic for azathioprine rises from zero in 1980 to more than 50 percent in the mid-1980s before declining to 12 percent by 1992 .

Table 11.4 summarizes prescriptions for each drug for which the primary diagnosis was RA. The total number of mentions is greater than the number of visits because patients may be given more than one drug per visit (this occurs in approximately 80 percent of visits). Since these data are compiled from simple counts of mentions and thus do not reflect differences in the size of prescriptions, these are approximations at best. The share of gold salts, for example, may well be overstated because patients

Table 11.3

NDTI Drug Mentions by Diagnosis: Fraction of RA by Drug

\begin{tabular}{lcccl}
\hline Drug & $\begin{array}{c}1980 \\
(\%)\end{array}$ & $\begin{array}{c}\text { Mean } \\
(1980-92 \%)\end{array}$ & $\begin{array}{c}1992 \\
(\%)\end{array}$ & $\begin{array}{c}\text { Major Other Use } \\
\text { (Mean 1980-92, \%) }\end{array}$ \\
\hline Auranofin & & 97 & 84 & \\
Azathioprine & 92 & 86 & 12 & Transplant, 56 \\
Gold salts & 0 & 91 & 91 & \\
Antimalarials & 92 & 64 & 56 & Circulatory disorders, 9 \\
Methotrexate, injectable & 79 & 21 & 22 & Cancer, 59 \\
Methotrexate, oral & 0 & 65 & 69 & Skin disease, 17 \\
D-penicillamine & 91 & 81 & 72 & Digestive disorders, 83 \\
Sulfasaiazine & 0 & 8 & 11 &
\end{tabular}

Introduced in 1985.

Table 11.4

Share of NDTI Drug Mentions for RA (Mean 1980-94)

\begin{tabular}{lc}
\hline Drug Category & Share of Mentions $(\%)$ \\
\hline Cortisone & 19.8 \\
Other & 14.6 \\
NSAIDs & 36.2 \\
DMARDs & 29.4 \\
Auranofin & 4.7 \\
Azathioprine & 4.5 \\
Gold salts & 37.3 \\
Antimalarials & 17.4 \\
Methotrexate & 23.7 \\
D-penicillamine & 9.4 \\
Sulfasalazine & 2.6 \\
\hline
\end{tabular}




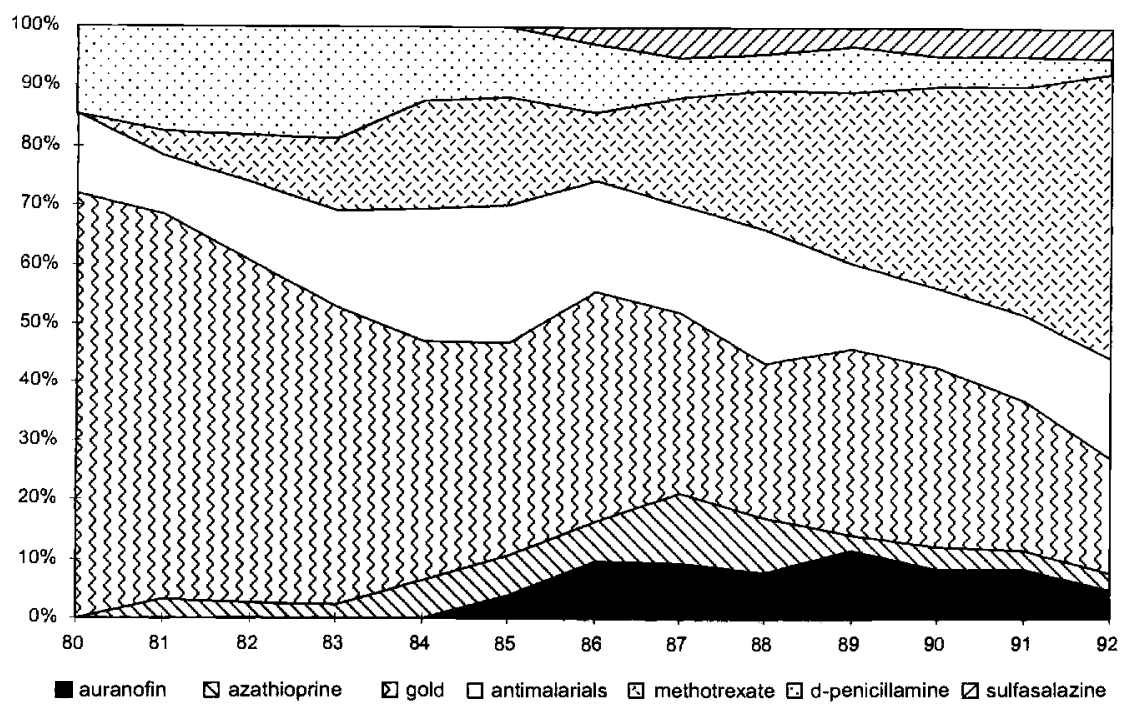

Fig. 11.2 DMARDs: share within class of NDTI mentions for RA

make weekly visits to their physician for an injection. On the other hand, methotrexate may be prescribed in a more traditional manner with the patient visiting the physician (and obtaining a new or refill prescription) much less frequently. Nonetheless these fractions are our best estimate of each drug's share of the RA market. ${ }^{\text {' }}$

Figure 11.2 summarizes quantity shares within the DMARD market graphically. The total size of the DMARD market (as measured by the number of mentions of these drugs in the NDTI grew somewhat over time from about 1.9 million mentions per year in the early 1980 s to around 2.4 million in the early 1990s. Much of this growth was driven, however, by increases in the numbers of patients diagnosed with $\mathrm{RA}$, which reflect changes in the demographics of the U.S. population. DMARDs as a class were a somewhat larger share of the total RA market at the end of our sample period (around 28 percent in 1992 compared to 21 percent in 1980) which may reflect some market-expanding effect of improved quality and new product introductions, but these changes are dominated by movements within the DMARD market. The most striking feature of figure 11.2 is the substantial fall in the share of injectable gold and the rise in the share of methotrexate. D-penicillamine's share falls steadily over time while the other drugs' shares are relatively small and stable. These patterns

9. As an alternative to using NDTI data we have explored a small data set constructed from the B. C. Pharmacare database where we include only prescriptions written by rheumatologists. The shares of different DMARDs in these data are very similar to those in the NDTI, but the data cover a somewhat shorter time period. 
reflect the general impression to be gained from reading the clinical literature: An increasing tendency to use methotrexate instead of gold, with mixed opinions about the therapeutic value of the other agents. Auranofin, the only new chemical entity to enter this market in the sample period, was launched in the mid-1980s as an orally administered alternative to the injectable gold compounds, but achieved only a modest 10 percent share.

To examine the relationship of prices and quantities to measured quality more carefully, we turn next to our estimation results.

\subsection{Results}

\subsubsection{Price Equation}

Table 11.5 reports results from estimating the reduced form hedonic price equation (1) using data on U.S. wholesale prices. To the extent that we expect relative prices to respond to changes in measured characteristics, the results are disappointing. In models 1 and 2 we regress the $\log$

Table 11.5

OLS Results: U.S. Wholesale Prices, 1980-92

\begin{tabular}{|c|c|c|c|c|c|}
\hline & Model 1 & Model 2 & Model 3 & Model 4 & Model 5 \\
\hline Constant & $4.84(0.94)$ & $-0.14(0.14)$ & $-1.10(0.14)$ & $-0.12(0.79)$ & $-0.77(0.17)$ \\
\hline Efficacy & $-5.68(1.03)$ & & & $-1.11(0.81)$ & \\
\hline Toxicity ${ }^{b}$ & $1.15(0.38)$ & $0.82(0.41)$ & & $0.55(0.26)$ & $0.83(0.22)$ \\
\hline $\begin{array}{l}\text { Improvement } \\
\text { in GS }\end{array}$ & & $-0.44(0.42)$ & & & $-1.37(0.22)$ \\
\hline $\begin{array}{l}\text { Improvement } \\
\text { in } \mathrm{TJC}\end{array}$ & & $-1.22(0.40)$ & & & $0.22(0.29)$ \\
\hline $\begin{array}{l}\text { Improvement } \\
\text { in ESR }\end{array}$ & & $0.39(0.38)$ & & & $-0.36(0.23)$ \\
\hline Dummy 1981 & & & 0.11 & 0.10 & 0.04 \\
\hline Dummy 1982 & & & 0.30 & 0.26 & 0.09 \\
\hline Dummy 1983 & & & 0.51 & 0.44 & 0.23 \\
\hline Dummy 1984 & & & 0.66 & 0.59 & 0.41 \\
\hline Dummy 1985 & & & 0.83 & 0.77 & 0.72 \\
\hline Dummy 1986 & & & 0.95 & 0.88 & 0.82 \\
\hline Dummy 1987 & & & 1.03 & 0.96 & 0.89 \\
\hline Dummy 1988 & & & 1.17 & 1.10 & 0.78 \\
\hline Dummy 1989 & & & 1.23 & 1.14 & 1.12 \\
\hline Dummy 1990 & & & 1.34 & 1.22 & 1.24 \\
\hline Dummy 1991 & & & 1.44 & 1.32 & 1.34 \\
\hline Dummy 1992 & & & 1.48 & 1.36 & 1.38 \\
\hline$R^{2}$ & 0.29 & 0.24 & 0.72 & 0.74 & 0.84 \\
\hline
\end{tabular}

Note: Standard errors in parentheses; $N=78$. Dependent variable: Current U.S. dollars per daily dose. Efficacy $=(1-$ dropout rate for lack of efficacy $)$.

'Toxicity $=$ dropout rate for toxicity. 
of daily dose price on to characteristics variables alone. The estimated parameters are contrary to our prior beliefs: Efficacy (whether measured by the fraction of patients who do not drop out of trials because of lack of efficacy, or by changes in the physiological measurements GS, TJC, and ESR) is negatively associated with price, while toxicity is positively associated. These results do not change when we add a set of year dummies to the list of explanatory variables, though the fit of the equation improves markedly. Coefficients on the time dummies imply a steady upward movement in prices, which is very similar whether or not we attempt to control for quality change.

Why the characteristics variables should perform so poorly is puzzling. Considerable experimentation with alternative ways of computing these quality measures from the clinical trials data did not improve these results. Regardless of whether efficacy and toxicity are measured relative to placebo or as unadjusted changes, or which of the alternative weighting and updating schemes discussed above is used, we still obtain the "wrong" signs on the estimated coefficients. One reason may be that measurement error is biasing the coefficient estimates. ${ }^{10}$ This possibility should be taken seriously as the clinical trials literature is not unambiguous, and prescribing physicians may be unaware or skeptical of the results reported in the studies we use here.

We prefer, however, to interpret these findings as evidence of an alternative hypothesis about the nat ure of this market. Given the serious medical situation of most patients who are given DMARDs, and their lack of alternatives, it seems likely that demand is quite inelastic. With many of these drugs having their primary use elsewhere, it is plausible that their prices are essentially exogenous to the RA market. Casual inspection of the raw data suggests that the level of prices for these drugs bears little relationship to measured characteristics, with some of the most toxic and least efficacious drugs having the highest prices. Furthermore, looking at changes over time, we see that prices for most of the drugs move steadily upward with general inflation, with little change in relative prices. Where movements in prices conform to our priors, any correlation with changes in measured quality appears to be swamped by the rest of the data. Seen in this light, the results obtained in the price equation may simply be spurious, with the coefficients on the characteristics variables reflecting confounding with other factors determining prices.

Experience with other pharmaceutical price data as well as informal evidence gathered in discussion with industry executives and other experts suggests also that relative prices for these products are very sticky. There is some evidence for sensitivity of pharmaceutical prices to exogenous

10. Recall that with more than one variable potentially mismeasured, the resulting bias on estimated coefficients is hard to predict and need not always be toward zero. 
shocks such as regulatory changes; see Anis and Wen (1998) and Scott Morton (1997). On the other hand, other studies have found prices of branded products to be remarkably insensitive to patent expiration and large-scale entry by generics (see Griliches and Cockburn 1995). It may not therefore be a gross mischaracterization of historical industry pricing practice to summarize it as setting prices once (in real terms) at the time of product launch, with subsequent revisions limited largely to adjustments for general inflation applied across a producer's entire product line. Any price premium related to improved quality will therefore be difficult to see except in markets with substantial numbers of new products entering over time, which is not the case here. In this light, it is worth pointing out that poor results were also reported in Berndt and Finkelstein's (1992) study of hedonic pricing of anti-hypertensives, another drug class with a low rate of substantively new chemical entities reaching the market.

With prices set exogenously to this market, and given that relative prices are likely far down the list of patients' and physicians' concerns when choosing which drug to use, it is likely that the impact of changes in quality manifest themselves in the market largely in changes in quantities, which is where we turn next.

\subsubsection{Market Share Equations}

Table 11.6 presents estimates of the parameters of equation (5), with and without fixed drug effects. In models 6 and 8 the toxicity and efficacy

Table 11.6

OLS Results: Quantity Share Regression, 1980-92

\begin{tabular}{lcrrr}
\hline & $\begin{array}{c}\text { Model 6 } \\
\text { (latest) }\end{array}$ & $\begin{array}{c}\text { Model 7 } \\
\text { (MA) }\end{array}$ & $\begin{array}{c}\text { Model 8 } \\
\text { (latest) }\end{array}$ & $\begin{array}{c}\text { Model 9 } \\
\text { (MA) }\end{array}$ \\
\hline Constant & $-18.81(3.47)$ & $-15.73(3.58)$ & $-8.63(2.15)$ & $-8.50(2.13)$ \\
Efficacy $^{*}$ & $8.94(3.73)$ & $4.87(4.04)$ & $-0.94(2.12)$ & $-1.70(2.27)$ \\
Toxicity $^{\circ}$ & $-0.15(0.91)$ & $-0.53(0.92)$ & $-4.48(1.59)$ & $-4.11(1.55)$ \\
Improvement in GS & $1.59(0.85)$ & $2.48(1.25)$ & $0.38(0.47)$ & $0.00(0.67)$ \\
Improvement in ESR & $0.92(0.72)$ & $2.53(0.99)$ & $-0.41(0.49)$ & $1.23(0.94)$ \\
Price & $-0.43(0.61)$ & $0.09(0.68)$ & $0.30(0.48)$ & $0.36(0.48)$ \\
Dummy auranofin & & & $-0.48(0.46)$ & $-0.33(0.46)$ \\
Dummy azathioprine & & & $-0.52(0.33)$ & $-0.15(0.39)$ \\
Dummy gold salts & & & $2.36(0.28)$ & $2.34(0.27)$ \\
Dummy antimalarials & & & $-0.09(0.51)$ & $0.14(0.50)$ \\
Dummy methotrexate & & & $1.41(0.31)$ & $1.63(0.34)$ \\
Dummy & & & $0.71(0.31)$ & $0.58(0.30)$ \\
$\quad$ d-penicillamine & & 0.29 & 0.79 & 0.80 \\
$R^{2}$ & 0.26 & & & \\
\hline
\end{tabular}

Note: Standard errors in parentheses; $N=78$. Dependent variable: Current U.S. dollars per daily dose. ${ }^{a}$ Efficacy $=(1-$ dropout rate for lack of efficacy $)$.

${ }^{\mathrm{b}}$ Toxicity $=$ dropout rate for toxicity. 
variables are based only on the most recently published trial in each year, while in models 7 and 9 they are computed as a three-year moving average of published trial results. In both cases the drug effect is calculated relative to placebo, but very similar results are obtained using just the change relative to the baseline values.

Results in models 6 and 7 are encouraging. The signs of the coefficients on the characteristics variables conform to our priors, with increased toxicity negatively associated with market share, and increased efficacy positively associated. Though the coefficient on price is insignificant, and corresponds to a very small elasticity, it is at least negative in model 6 . A very small price effect is also consistent with our interpretation of results from estimating the price equation.

Models 8 and 9 include fixed drug effects in the estimation to control for drug-specific problems in measuring market share or characteristics. Several of these dummies are highly significant, and they markedly improve the fit of the model, suggesting that we do indeed have systematic problems in measuring market shares. Furthermore the estimated coefficients on the other variables change substantially when we include fixed drug effects, indicating that the equations omit significant variables driving quantities consumed, either quality characteristics of drugs or other drugspecific factors which determine demand.

\subsection{Conclusion}

Economic considerations appear to play a relatively minor role in the market for DMARDs. Information from published clinical trials relating to key quality characteristics of these drugs (efficacy and toxicity) is statistically associated with changes in their quantity shares in this market, but has no consistent impact on relative prices. Given the nature of RA, these results may not be too surprising. They do, however, point to some interesting economic issues which we have not attempted to address in this study.

First, there is the question of using prices to measure the impact of technical change on consumer welfare in markets such as this one. Most prior work on innovation, quality change, and pricing has examined the prices of new goods which embody technological change in the form of improvements to tangible aspects of quality. Here the technical change takes a rather unusual form: $R \& D$ generates revisions to the intangible information set possessed by physicians and patients, affecting perceived quality rather than physical characteristics such as speed, durability, weight, and so on. R\&D surely improves welfare in this context, but the fact that relative prices in this market change very little (and are most likely determined exogenously) and that demand appears to be quite price inelastic means that its impact is very difficult to see in price space. Rather, 
the most visible direct effect of changes in quality is seen in movements in quantities, which has significant implications for how we should interpret movements in, for example, a fixed-weight price index.

Second, these results hint at an interesting variety of nonprice competition. Rents to producers in this market are determined initially by the level of prices (which to a rough approximation they set once in real terms, often based upon conditions prevailing in unrelated markets) and then by the evolution of quantities as consumers and/or their agents respond to exogenous changes in perceived quality. In such circumstances the role played by marketing and promotional activity may well be very important. Our analysis here is based on the generation of new information about product quality in the form of publication of research results in peer reviewed journals by (hopefully) impartial authors. The question of how this information reaches practicing physicians and their patients has not been examined here. In future work we hope to extend our analysis of this market to include marketing and promotional activity by producers of these drugs, which may shed light on the interesting question of the relative importance of objective versus persuasive information in drug choices.

\section{References}

Anis, A. H., and Q. Wen. 1998. Price regulation of pharmaceuticals in Canada. Journal of Health Economics 17 (1): 21-38.

Berndt, E. R., I. M. Cockburn, and Z. Griliches. 1997. Pharmaceutical innovations and market dynamics: Tracking effects on price indexes for antidepressant drugs. Brookings Papers on Economic Activity, Microeconomics, 133-88.

Berndt, E. R., and S. N. Finkelstein. 1992. Price indexes for anti-hypertensive drugs that incorporate quality change: A progress report on a feasibility study. MIT Program on the Pharmaceutical Industry, Working Paper no. 6-92.

Berry, S. B. 1994. Estimating discrete-choice models of product differentiation. RAND Journal of Economics 25:242-62.

Berry, S. B., J. Levinsohn, and A. Pakes. 1995. Automobile prices in market equilibrium. Econometrica 63:841-90.

Brewerton, D. 1994. All about arthritis. Cambridge, Mass.: Harvard University Press.

Cash, J. M., and J. H. Klippel. 1994. Second-line drug therapy for rheumatoid arthritis. New England Journal of Medicine 330:1368-75.

Felson, D. T., J. J. Anderson, and R. F. Meenan. 1990. The comparative efficacy and toxicity of second-line drugs in rheumatoid arthritis: Results of two metaanalyses. Arthritis and Rheumatism 33:1449-59.

Griliches, A., and I. M. Cockburn. 1995. Generics and new goods in pharmaceutical price indexes. American Economic Review 84:1213-32.

IMS America Inc. 1980-94. National drug and therapeutic index-Drugs. Plymouth Meeting, Pa.: IMS America.

- 1980-94. National drug and therapeutic index-Diagnosis. Plymouth Meeting, Pa.: IMS America. 
King, C. 1996. Marketing, product differentiation, and competition in the pharmaceutical industry. MIT Program on the Pharmaceutical Industry, Working Paper no. 39-96.

Medical Sciences Bulletin. 1994. Focus on rheumatoid arthritis. Levittown, Pa.: Pharmaceutical Information Associates.

Pincus, T., and L. F. Callahan. 1993. The "side-effects" of rheumatoid arthritis: Joint destruction, disability and early mortality. British Journal of Rheumatology 32 (suppl.): $28-37$.

Reynolds, D. L., et al. 1993. Modelling the population health impact of musculoskeletal diseases. Journal of Rheumatology 20 (6): 1037-47.

Scott Morton, F. 1997. The strategic response by pharmaceutical firms to the Medicaid most-favored-customer rules. RAND Journal of Economics 28: 269-90.

Steinman, L. 1993. Autoimmune disease. Scientific American 269 (3): 106-14.

Suslow, V. 1992. Are there better ways to spell relief? An hedonic pricing analysis of ulcer drugs. School of Business Administration, University of Michigan Working Paper no. 696.

- 1996. Measuring quality change in the market for anti-ulcer drugs. In Competitive strategies in the pharmaceutical industry, ed. R. Helms. Washington D.C.: American Enterprise Institute.

Torrance, G. W., and D. Feeny. 1989. Utilities and quality-adjusted life years. International Journal of Technology Assessment in Health Care 5:559-75.

Wolfe, F. 1990. 50 Years of Antirheumatic Therapy: The Prognosis of RA. Journal of Rheumatology. 22 (suppl.): 24-32.

\section{Comment J. Steven Landefeld}

First, let me begin by saying that this is a good piece of research. It deals with an important problem in an interesting manner. As the authors point out, arthritis has a large economic impact, imposing a cost in the United States, for example, of over $\$ 65$ billion in medical treatment costs and lost wages and salaries associated with morbidity. The disease also has an important "quality" dimension aside from its impact on mortality in that its main effect is a reduction in quality of life for patients who experience significant pain and suffering.

The study represents an original application of hedonic analyses: Instead of examining a set of new goods or services with new or different characteristics, as most hedonic studies of quality change have (new generations of computers, new types of telecommunications equipment, new treatments for heart attacks), it looks at a mainly fixed set of drugs whose "perceived" characteristics change. Most of the drugs examined in this study have been in use for a long time; what has changed is the medical profession's assessment of their efficacy and toxicity.

The results of the study are also interesting. While quality (as measured

J. Steven Landefeld is director of the Bureau of Economic Analysis, U.S. Department of Commerce. 
by efficacy and toxicity) appears to affect the share of the market accounted for by the various drugs, there does not appear to be any discernible impact of quality differences on their prices relative to one another. Indeed, quality and price appear to be inversely correlated-in that higher prices are associated with lower efficacy and higher toxicity. In explaining this result, the authors point to the importance of other factors in determining prices, including the use of these same drugs to treat other diseases; inelastic demand by patients with rheumatoid arthritis (RA); institutional stickiness in prices; and other price-determining factors such as promotional expenditures.

As the representative of a U.S. statistical agency, I was particularly interested in what we at statistical agencies, along with other researchers, could learn from this study. The first lesson that I take away from this study is that hedonics may not always work. Hedonic regressions are an attempt to use observable differences in the qualities and characteristics of goods to see what consumers are willing to pay for those differences. Where those differences are hard to measure or where there are imperfections in markets that make prices insufficiently reflective of consumer preferences, hedonics may not work or may produce implausible results. Alternatively, perhaps we should interpret the results of this study as an example where the measured price increase for the product understates the real (qualityadjusted) price increase. That is, drug prices went up, but quality declined. However, in this case, the issue would appear to be mainly related to problems in measuring quality change and imperfections in the market for these drugs.

In addition to these general conclusions, there are a number of more specific lessons to be learned from this study. First, in setting up a study, one should avoid cases where prices are exogenously determined. As the authors point out, most of the drugs used in treating RA were developed for treating other diseases and a very large share of total demand for these drugs is for other diseases. As a result, changes in the perceived quality of the drugs in treating RA may have little impact on the price of the drug. It is difficult to assess the significance of this problem because of difficulties in interpreting drug mentions in the National Drug and Therapeutic Index (NDTI) and changes in the share of mentions over time. However, for a number of the drugs, other diseases accounted for the majority of the therapeutic drug mentions.

Second, one should be aware of the degree to which the price of the good or service in question is competitively determined and thus reflective of consumer preferences. It is only in competitive markets free of externalaties that we can presume that price is equated with marginal utility, which in turn is equated with marginal cost. If these conditions are not likely to be fulfilled, it is difficult to justify the use of hedonics to measure the value of changes in product quality. As economists and statisticians, we have no 
special expertise in estimating value and would rather observe through hedonics what consumers are willing to pay; however, if other factors have a large impact on market prices there is little justification for the use of the results from hedonic regressions.

In the case of drugs, there are significant problems fulfilling the competitive requirements for hedonic analysis. The demand for the product is in great part determined by the physician rather than the consumer. Longlived patents, advertising, distribution networks, and physician incentives have frequently been cited as sources of market power in the determination of drug prices. In addition, the third-party payment system means that the price faced by the consumer is often below the market price. In 1995, for example, third-party payments covered about 40 percent of overall drug expenditures and the proportion for prescription drugs such as those studied in this paper are probably higher.

The third thing that we learn from this study is the importance of having a good measure of quality (or of measuring a product whose quality actually changes). Most of the drugs used to treat RA have been in use for a long period of time and it seems unlikely that the quality of these drugs (as measured by efficacy or toxicity) changed over time. However, the measure of quality used in the study - perceived quality by physicians as a result of successive results from clinical trials-did change. The results from the study may simply reflect the fact that from the patient's viewpoint there were no changes in underlying quality and thus there was no little or no impact on price.

Indeed, the results of the study may fit expectations - with both market share and prices increasing with quality improvements-for the two drugs that are considered new. The first of these is Rheumatrex, which is not really a new drug, but was originally developed (under the name methotrexate) for use in the treatment of cancer. As the authors point out, Lederle gained regulatory approval for a formulation of methotrexate specifically targeted to the RA market and introduced it under the brand name Rheumatrex in 1986. Rheumatrex's price has increased more quickly than those of other drugs in the period following its introduction. During the study period, 1980-92, Rheumatrex's share of the market for RA drugs rose from 0 to 50 percent and appears to have accounted for the decline in the share of other drugs. The only other drug whose share has risen was auranofin, another new drug. Although the paper does not present separate quality-adjusted results for these drugs, the existing results suggest that one should concentrate on new products where characteristics are changing. If the characteristics of the other, older drugs really didn't change, the results - that the coefficients with respect to the effect of quality on price were mainly negative and insignificant - should not be surprising. And the apparent relationship between quality and market shares for 
the broad group of drugs may be a spurious correlation driven by the rising share accounted for by Rheumatrex.

Given this list of things to watch out for in future studies, what advice can we offer the authors and other researchers? The authors of this study might profitably concentrate on methotrexate and auronafin. They might also develop an expanded joint product model for these two drugs that examines their other uses and the effect of promotional efforts on their prices and market shares.

With respect to other researchers about to embark on hedonic analyses, they might profit when deciding on a subject for study by examining the list of factors listed above (exogenously determined prices, serious imperfection in markets, and inadequacies in measure of quality change). Researchers may also want to examine the data a bit, including quick reviews of simple correlations, before they commit to a particular project.

If a number of these problems are present, the researcher faces the choice of moving on to another research project or seeing what useful information can be extracted from the study. Researchers should be careful not to move on to another project too quickly. Moving on not only risks ignoring an important issue (either in terms of economic importance or in terms of social policy), but may bias the results by ignoring those cases where quality has gone down. Thus, work on important issues should not be too quickly discarded even with the presence of the problems discussed above, but should be considered and where feasible work should proceed sequentially with each step having varying degrees of immediate application.

One could, for example, begin by using the IMS and NDTI data in this study to develop a drug services price index that would measure the changing cost of drug therapy for treating a case of rheumatoid arthritis. Given the importance of this disease, such an index would be most useful even if a hedonic quality-adjusted price index were not available. One could probably also use the annual price information and market share information to create an annual-weighted Fisher index to avoid the bias that would be introduced into a fixed-weighted price index by the increasing market share of Rheumatrex. Finally, as suggested above, by focusing on the new drugs Rheumatrex and auranofin, and enriching the model to consider other factors influencing price and market share, one might develop a hedonic quality-adjusted index. 
This Page Intentionally Left Blank 